C-A/AP/\#161

August 2004

\title{
HOM COUPLER MEASUREMENT AND SIMULATION
}

\author{
Yongxiang Zhao and Harald Hahn
}

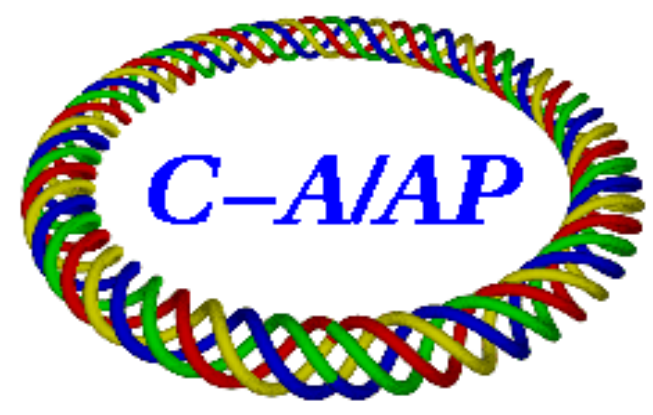

Collider-Accelerator Department Brookhaven National Laboratory Upton, NY 11973 


\title{
HOM COUPLER MEASUREMENT AND SIMULATION
}

\author{
Yongxiang Zhao and Harald Hahn \\ Brookhaven National Laboratory, Upton, NY 11973
}

\section{INTRODUCTION}

Higher order mode (HOM) couplers have been widely used in superconducting cavities to absorb the HOM energy stimulated by the beam. However, overheating of the coupler becomes a concern when the beam current is strong. For example, the ERL project (Energy Recovery Linac) requires average current of $500 \mathrm{~mA}$. To this end, it is interesting to know the current distribution at the HOM coupler. In this note, the procedure how to estimate the current on the HOM coupler is addressed.

As first step, network analyzer measurements were made with the HOM coupler mounted on a coaxial cable test setup. PSpice was then applied on an equivalent circuit to simulate the measured results with the parameters adjusted in such a way as to get good agreement. Subsequently, the excitation field at the HOM coupler in the test setup was calculated and compared with the field on the beam pipe of a 5 cell cavity. Finally, the current magnitude on the coupler was defined in relation to the accelerating gradient.

\section{TEST SET-UP AND HOM COUPLER}

Fig. 1 shows the schematic of the test set-up. The main body of the set-up is a coaxial line, onto which the HOM coupler was mounted. Both the line and the coupler are connected to a network analyzer (Agilent 8753ES) for measuring the forward scattering coefficients at the HOM output. The outer and inner diameters of the coaxial line are 5.75 and $1 / 4$ in. respectively, which correspond to a characteristic impedance of $188 \Omega$. The connecting cables of the network analyzer are of $50 \Omega$. In order to match the coaxial line impedance to the $50 \Omega$ connecting cables of the network analyzer matching resistances are inserted as shown in the figure.

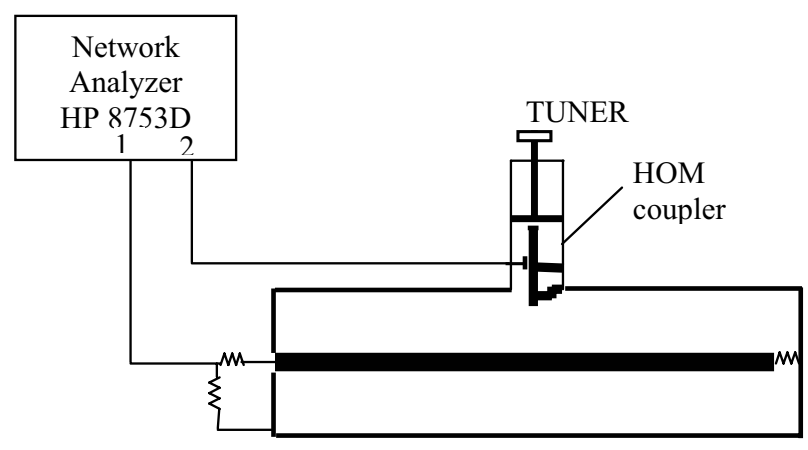

Fig.1. Schematic of the test set-up with coaxial line and HOM tuner 


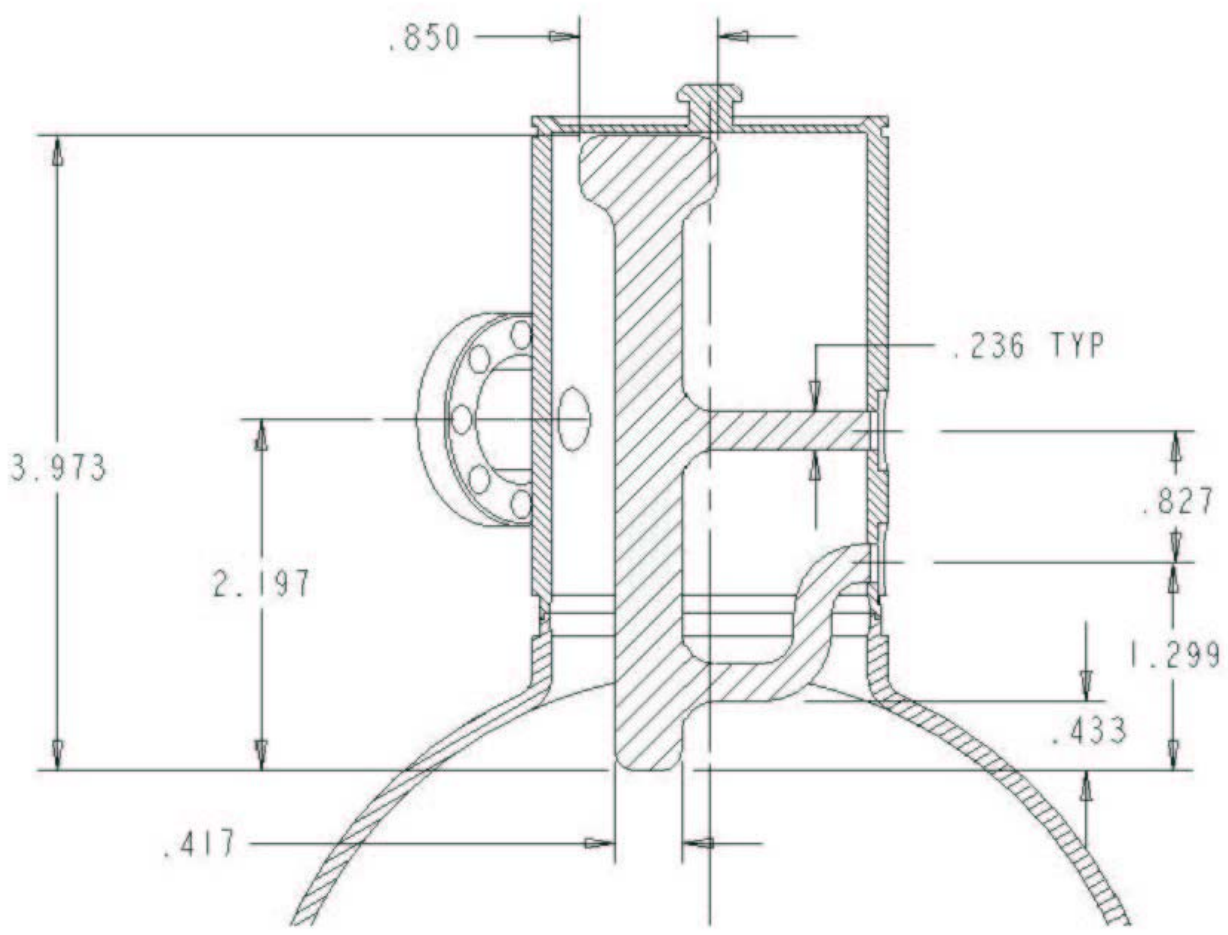

Fig. 2. Details of the HOM coupler and its installation

Construction details and dimensions of the installed HOM coupler are shown in Fig. 2. The figure schematically shows the outer envelop (i.e. the cylinder), the tuner at the top, and the output coupling port at the left. The dimensions of the HOM coupler shown are in inches. The diameter of the inner surface of the cylinder is $50 \mathrm{~mm}$. The tuner on the top part is adjustable up and down. The output is coupled by a capacitance between it and the inner conductor. The protrusion of the tip of the HOM coupler into the coaxial line is $0.917^{\prime \prime}(23.3 \mathrm{~mm})$.

\section{RESULTS OF THE MEASUREMENT}

The purpose of the HOM coupler is to absorb as much as possible the HOM energy stimulated by the beam. Meanwhile it should absorb as little as possible the fundamental mode energy in the cavity. To this end, the measurement of S21 with the above set-up is expected to have a minimum (a notch) at the fundamental frequency, and to have rather large values at higher frequencies. This is achieved by the adjustment of the tuner. Fig. 3 shows a typical S21 response, with its amplitude at the top of the figure and its phase at the bottom.

Note that the "notch" is at $707 \mathrm{MHz}$ with S21 of $-100 \mathrm{~dB}$ and is very sharp. S21 at higher frequencies is around $-20 \mathrm{~dB}$, or $80 \mathrm{~dB}$ higher than that of the fundamental frequency. However, the curve at high frequency looks irregular. It is believed to be due to the system itself. Fig. 4 shows the response of the set-up with the HOM coupler being replaced by a simple probe. The irregularity is due to imperfect matching. 


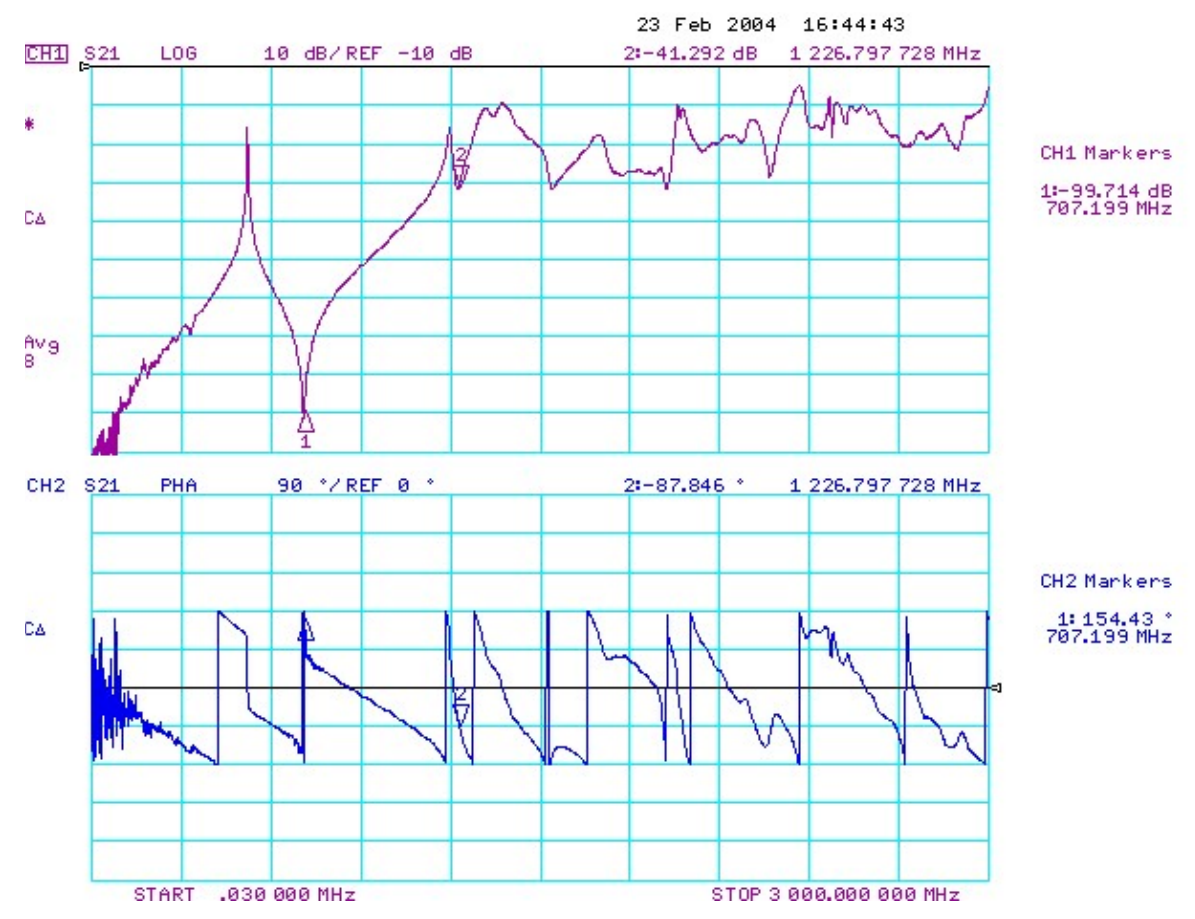

Fig. 3. The measured S21frequency response via network analyzer Agilent 8753ES, note the "notch" is at $707 \mathrm{MHz}$. The curve on the bottom is its phase.

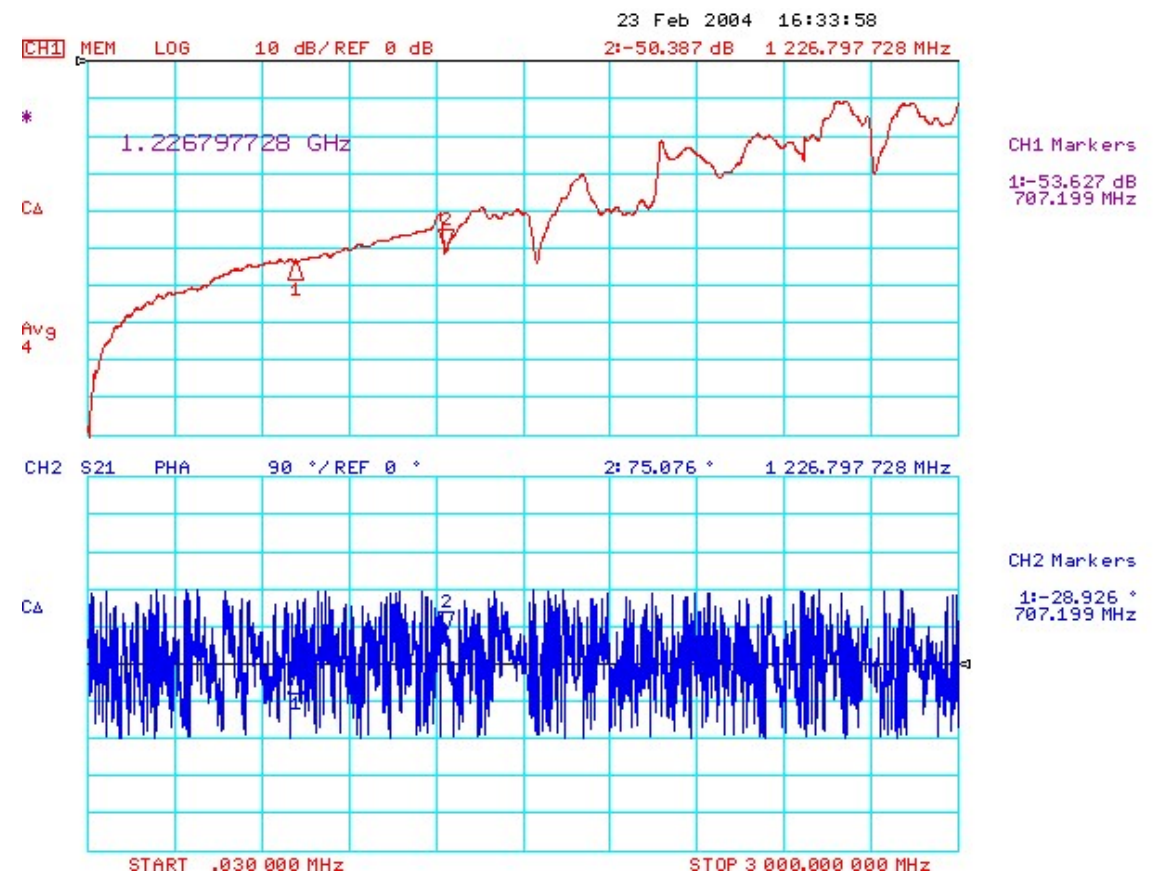

Fig. 4 The response of the set-up itself without HOM coupler. 


\section{THE SIMULATION PER PSpice}

The HOM coupler structure shown in the Fig. 2 can be regarded as a semi-lumped circuit shown in Fig. 5. The two sections of the inner conductor are represented by two short piece of transmission lines $\mathrm{T} 1$ and $\mathrm{T} 2$, while the two branches are represented by lumped inductances L1 and L2. The tuner is a variable capacitance C4. The output cable is coupled to the inner conductor via a capacitance C3 with a parasitic inductance L3.

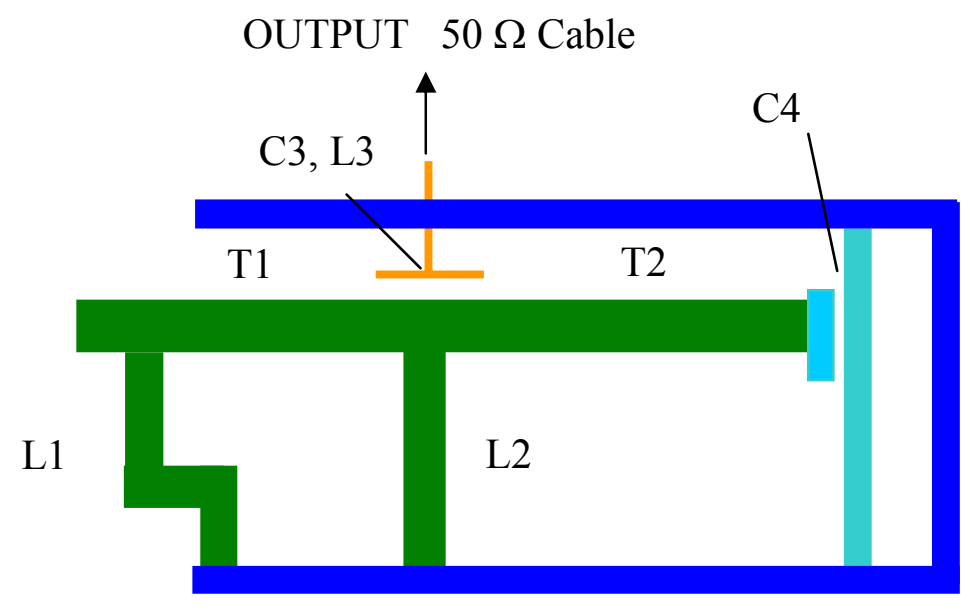

Fig. 5. The analogy model of the HOM coupler

The values of the circuit elements are difficult to calculate precisely. Nevertheless a rough estimate would be good enough, provided that the final result is in agreement with the measurement. According to the structure of the HOM coupler, the transmission line is composed of eccentric cylinders with an outer diameter of $50 \mathrm{~mm}$, and an inner equivalent diameter of $11.9 \mathrm{~mm}$ with eccentricity of $10.1 \mathrm{~mm}$. One can then calculate the characteristic impedance to be about $75 \Omega$.

Based on the circuit concept, Fig. 6 renders a circuit model of the whole assembly. The simulated result from PSpice is shown in Fig. 7. As can be seen the shape is similar to the measured one shown in Fig. 3. The right part of Fig. 6 represents the HOM coupler, where T1 and T2 represent two sections of transmission line with characteristic impedance of $75 \Omega$ and length of 40 and $53 \mathrm{~mm}$ respectively. C3, L3 connects to a $50 \Omega$ resistance $\mathrm{R} 5$, which represents the output cable.

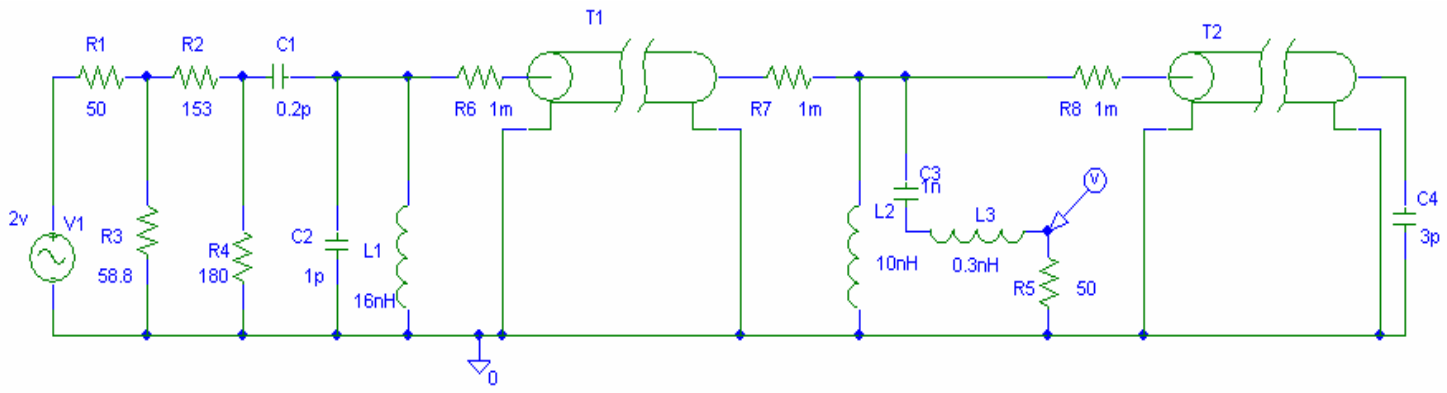

Fig. 6. The circuit model of the HOM coupler and the test assembly 


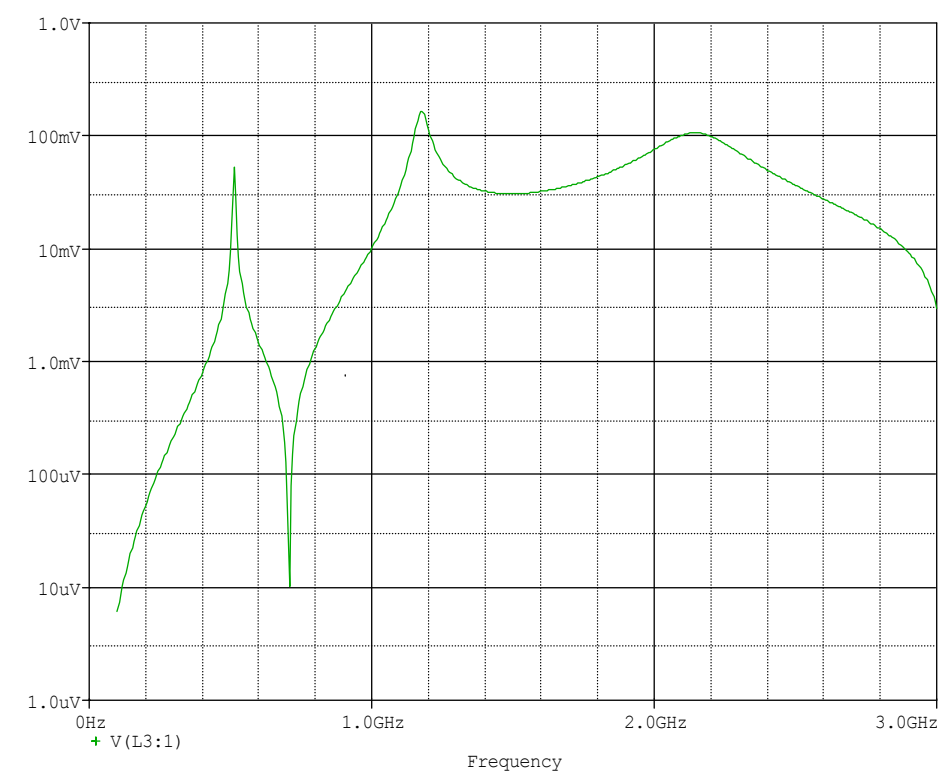

Fig. 7. The PSpice result response of the circuit shown in Fig. 6.

The voltage probe is at R5, namely the output voltage

The left part of Fig. 6 represents the test set-up. A normalized source voltage is defined as $2 \mathrm{~V}$, with an inner resistance R1 of $50 \Omega$, so that the output voltage of the source, i.e. the network analyzer, is $1 \mathrm{~V}$. The latter is used to calculate the scatter parameter S21. For example, if the output voltage (see Fig. 6) is $100 \mathrm{mV}$, then $\mathrm{S} 21$ is -20 $\mathrm{dB}, 10 \mu \mathrm{V}$ is $-100 \mathrm{~dB}$.

$\mathrm{R} 2$ and R3 form the matching network. R4 is the termination of the coaxial line. $\mathrm{C} 1$ represents the coupling between the inner conductor of the coaxial line and the HOM coupler.

One can see that inserted in the circuit are also three small resistances R6, R7, R8, which are not necessary for the simulation, but are necessary for checking the currents on the transmission lines. The results are shown next.

During simulation, the parameters have been adjusted in such a way as to best fit the measured results. The most important parameter is $\mathrm{C} 4$, adjustable by the tuner, to which the notch frequency is very sensitive. It is understandable, because $\mathrm{T} 2$ behaves as an inductance. At the notch frequency $\mathrm{f} 2$, T2 combined with $\mathrm{C} 4$ forms a series resonance, and it acts likes a short circuit.

There are two peak frequencies shown in the figure, namely fl about $510 \mathrm{MHz}$ and f3 about $1179 \mathrm{MHz}$, which are affected more or less by other parameters. The coupling capacitance $\mathrm{C} 1$ together with $\mathrm{C} 2$ are most important for the absolute value of the output.

Table 1 summarizes the most interesting data and gives the comparison between the measured and the simulated results at two peak and one notch frequencies. The good agreement verifies the circuit model and its chosen parameters. 
Table 1

\begin{tabular}{|c|c|c|}
\hline & Measured & Simulated \\
\hline $\mathrm{f} 1$ & $\sim 510 \mathrm{MHz}$ & $511 \mathrm{MHz}$ \\
\hline $\mathrm{A} 1$ & $\sim-25 \mathrm{~dB}$ & $-25.5 \mathrm{~dB}(53 \mathrm{MV})$ \\
\hline $\mathrm{f} 2$ & $707 \mathrm{MHz}$ & $709 \mathrm{MHz}$ \\
\hline $\mathrm{A} 2$ & $-99.7 \mathrm{~dB}$ & $-100 \mathrm{~dB}(10 \mathrm{uV})$ \\
\hline $\mathrm{f} 3$ & $\sim 1190 \mathrm{MHz}$ & $1179 \mathrm{MHz}$ \\
\hline $\mathrm{A} 3$ & $\sim-25 \mathrm{~dB}$ & $-15.7 \mathrm{~dB}(164 \mathrm{mV})$ \\
\hline
\end{tabular}

\section{THE CURRENT DISTRIBUTION ON THE COUPLER}

As mentioned above, one concern is the possible overheating of the HOM coupler. Thus it is interesting to know the current distribution. Since Fig. 6 has been verified to be correct, it is reasonable to use it to calculate the current. Fig. 8 and 9 show those PSpice results.

Fig. 8 shows the current versus frequency on both ends of T1 and T2. Fig. 9 shows the current on two branches as well as the output.

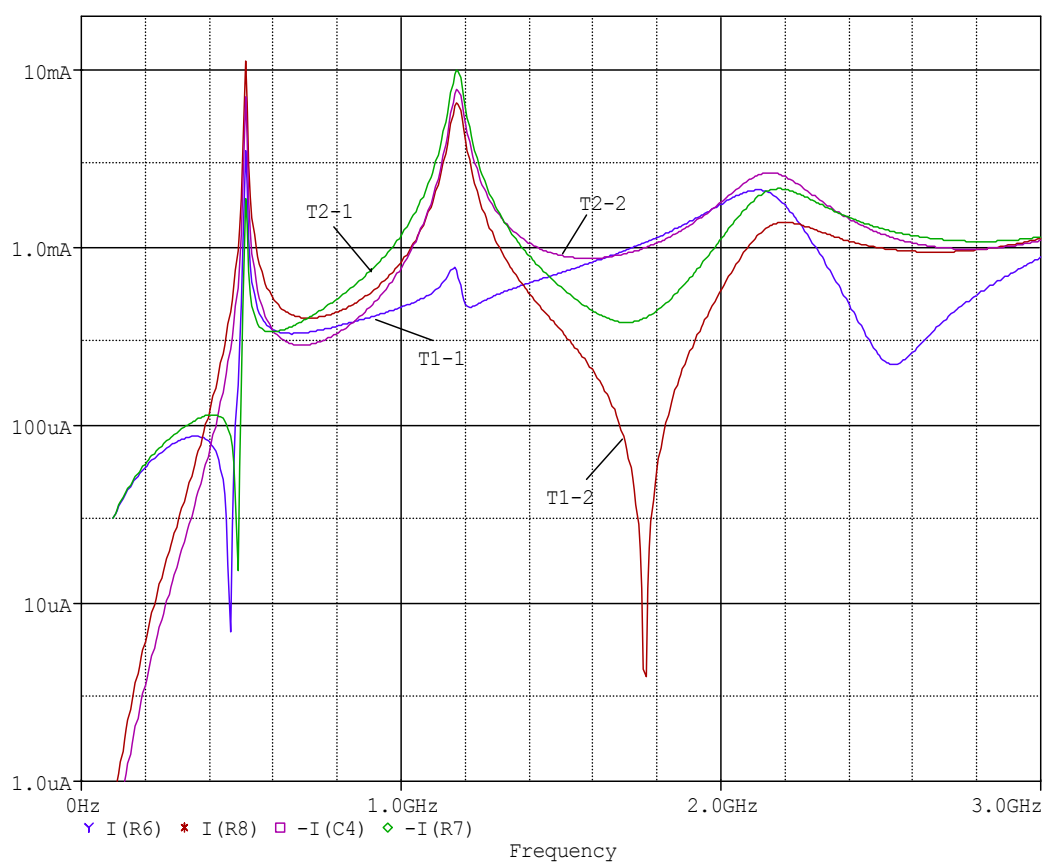

Fig. 8 The current versus frequency on both ends of T1 and T2 


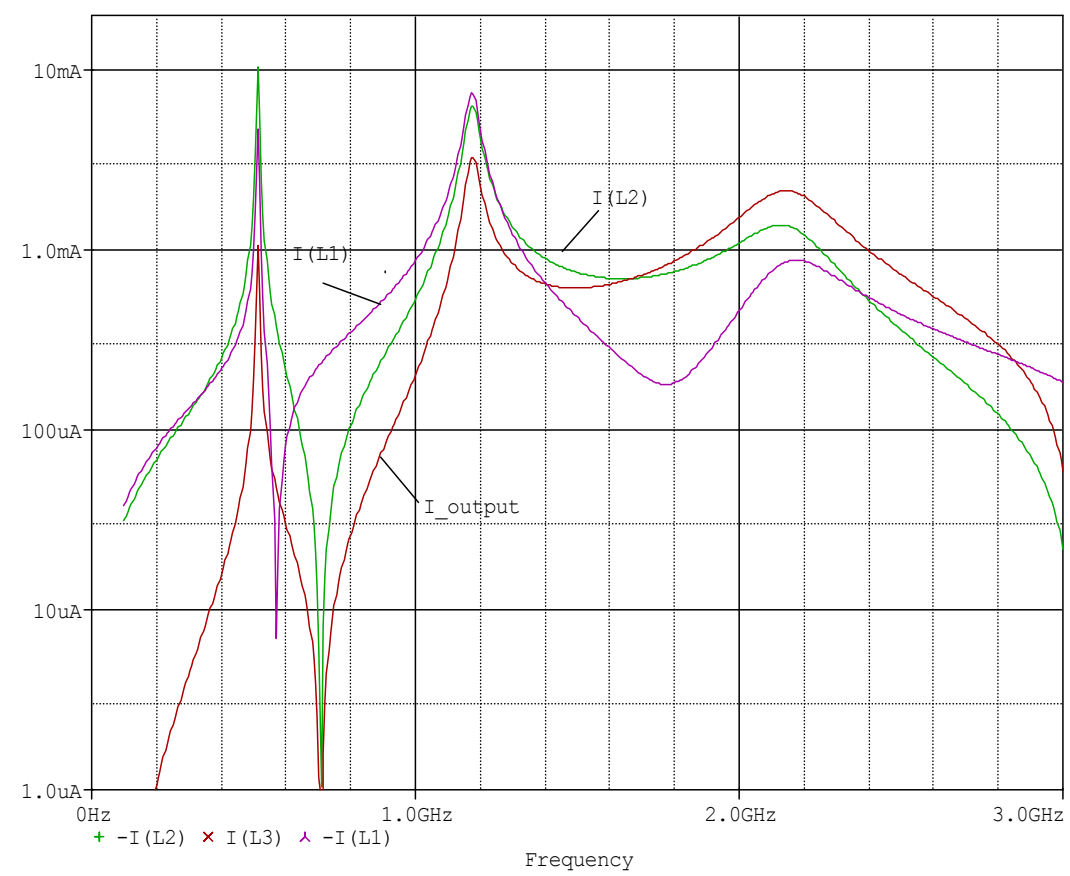

Fig. 9 The current versus frequency on L1, L2 and the output cable

Table 2 lists the current at different points and at the few most interesting frequencies. Of course, the greatest concern is that at the fundamental frequency. Listed are also the frequency $953 \mathrm{MHz}$ and $1173 \mathrm{MHz}$. The former is chosen because at earlier simulation by MAFIA we found the most dangerous high order mode to be around 950 $\mathrm{MHz}$. The latter is about the peak frequency in Figs. 7 and 8. The first peak of $510 \mathrm{MHz}$ can be ignored because it is below the fundamental and can not be harmful.

Table 2 The current at different points (all in $\mu \mathrm{A})$

\begin{tabular}{|c||c|c|c|c|c|c|c|}
\hline \multirow{2}{*}{ Points $\rightarrow$} & \multicolumn{2}{|c|}{ T1 } & \multicolumn{2}{c|}{ T2 } & \multicolumn{2}{c|}{ Branches } & Output \\
\hline Freq. $\downarrow$ & T1-1 (R6) & T1-2(R7) & T2-1(R8) & T2-2(C4) & L1 & L2 & R5 \\
\hline $709 \mathrm{MHz}$ & 334 & 402 & 401 & 284 & 236 & 1.03 & 0.201 \\
\hline $953 \mathrm{MHz}$ & 433 & 917 & 668 & 587 & 674 & 371 & 129 \\
\hline $1173 \mathrm{MHz}$ & 745 & 9900 & 6570 & 7740 & 7420 & 6320 & 3270 \\
\hline
\end{tabular}

The above data are based on a driver source of $1 \mathrm{~V}$. In order to find the corresponding data when the HOM coupler is inserted on a beam pipe, it is necessary to know the electric field at the tip of the coupler in the mentioned set-up.

At first one has to figure out the voltage on the cable. From Fig. 6 the coaxial line is equivalent with the circuit Fig. 10 and the voltage of the inner conductor is that at R4. 


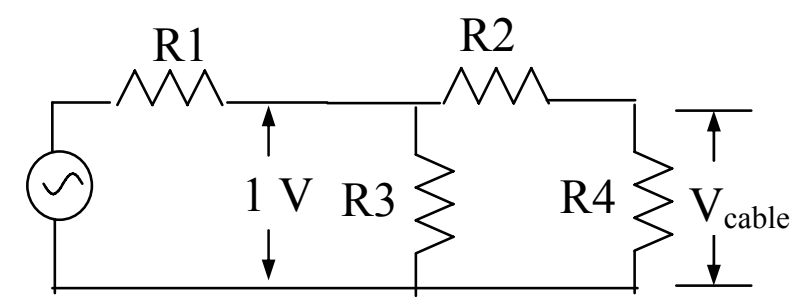

Fig. 10. The equivalent circuit of the coaxial cable assembly

Since the resistances R2 and R4 are known to be 153 and $180 \Omega$ respectively, and the voltage at the analyzer output is $1 \mathrm{~V}$ as mentioned above, the voltage on the inner conductor is readily calculated to be $0.54 \mathrm{~V}$.

The electric field at the surface of the outer conductor is related with the inner conductor voltage by:

$$
E\left(r_{2}\right)=\frac{V}{r_{2} \ln \left(r_{2} / r_{1}\right)}
$$

The diameters of the outer and inner conductor are $5.75 \mathrm{in}$. and $1 / 4 \mathrm{in}$. respectively. Substituting the known data, one finds the electric field at the tip of the HOM coupler as $2.36 \mathrm{~V} / \mathrm{m}$. This is the field that the data it Table 2 are based on.

\section{SUPERFISH SIMULATION}

The electric field at the location of the HOM coupler on the beam pipe of the SC cavity is obtained by simulation per SUPERFISH.

Fig. 11 shows the field pattern in the 5- cell cavity of the ERL. The top plot shows the entire field pattern while the bottom plot shows only the part beyond the cavity aperture.

Below the cut-off frequency, the fields are strongly dependent on the distance away from the cavity. Fig.12 shows the electric field along the beam pipe. $E_{z}$ is the longitudinal field at the axis. $E_{r}$ is the transverse field at the inner surface of the beam pipe, i.e. at $r=12 \mathrm{~cm}$, where the field is perpendicular to the surface and is the only component interacting with the HOM coupler. The longitudinal field at axis is not important in this case and for reference only. Because the fundamental frequency is below cutoff, the field decays exponentially, thus Fig. 12 shows straight lines in the logarithmic scale. 

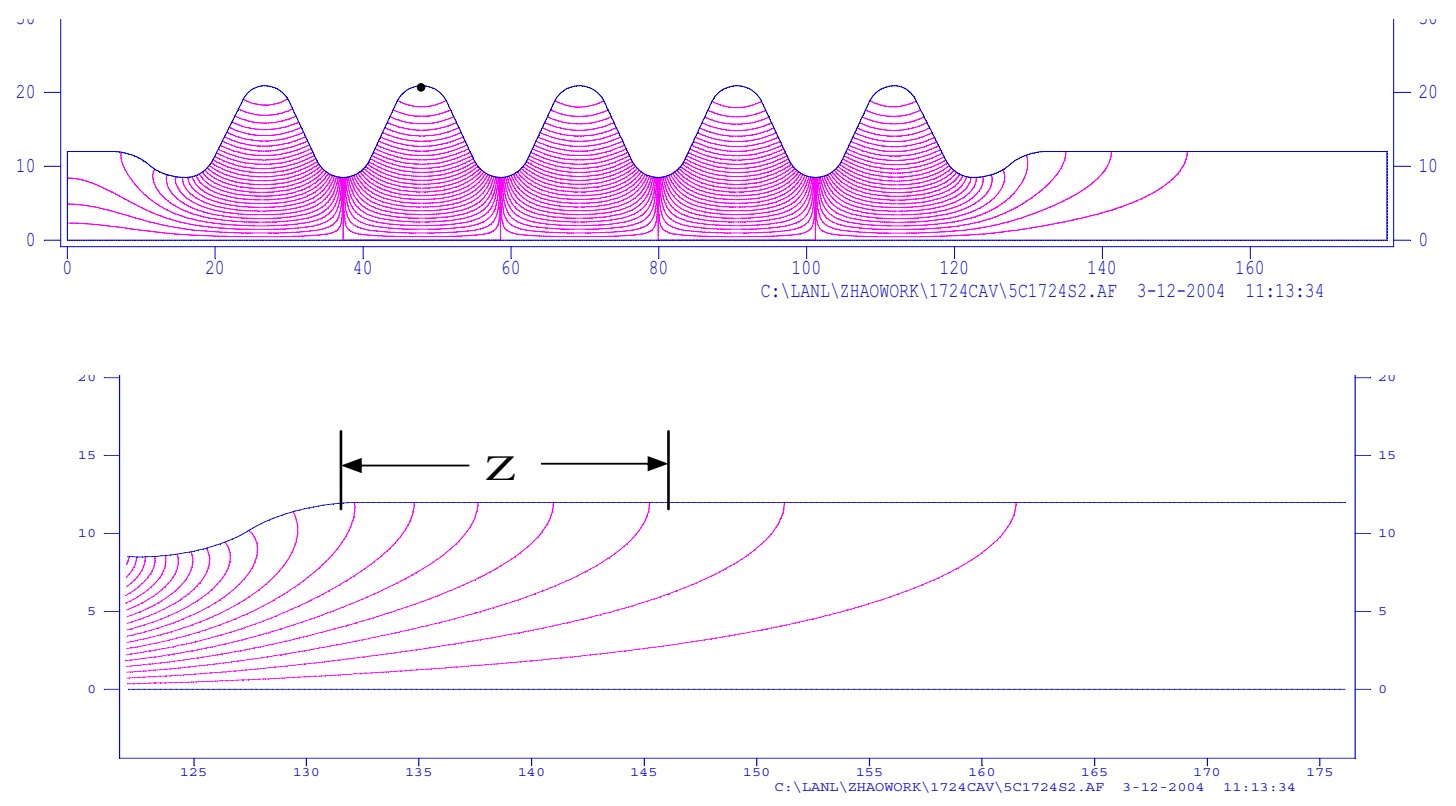

Fig. 11. The field pattern in the 5-cell superconducting cavity.

The bottom part shows the fields in the expanded right beam pipe.

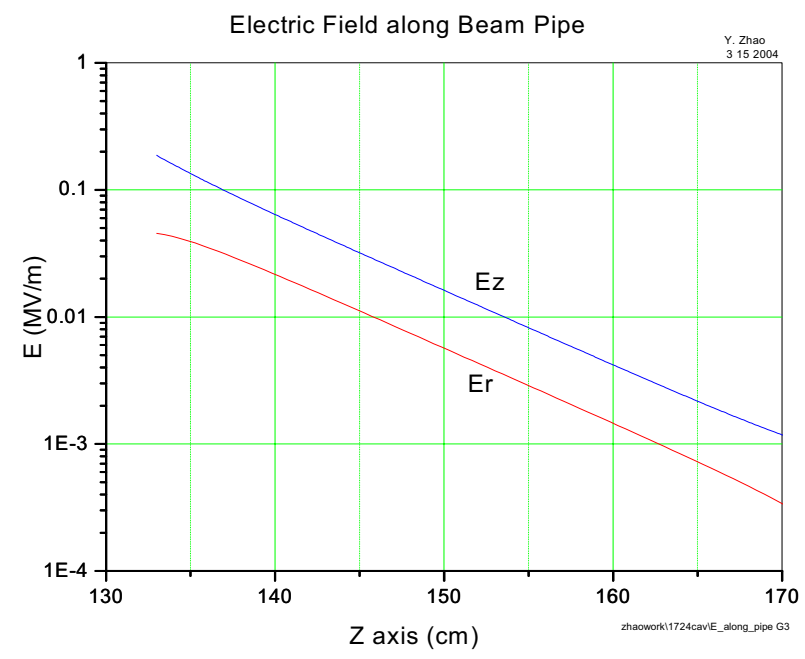

Fig. 12. Electric Field along the beam pipe

Of immediate interest is the absolute value of the field at a specified gradient. Note that the above data are based on the assumption that the average gradient is $1 \mathrm{MV} / \mathrm{m}$ and the total cavity length is $1.785 \mathrm{~m}$. Thus the total voltage of the 5 cell cavity is $1.785 \mathrm{MV}$.

It is convenient to change the normalization so that the whole cavity voltage is $1 \mathrm{MV}$, and define the location $z$ as the distance from the start point of the $24 \mathrm{~cm}$ diameter tube.

Table 3 lists the transverse field at different location. The left two columns show the original data, while the right two columns are normalized data. 
Table 3

\begin{tabular}{|c|c||c|c|}
\hline $\begin{array}{c}\text { Z-axis } \\
\mathrm{Cm}\end{array}$ & $\begin{array}{c}\mathrm{Er} \\
\mathrm{kV} / \mathrm{m}\end{array}$ & $\begin{array}{c}\text { Location } Z \\
\mathrm{~cm} .\end{array}$ & $\begin{array}{c}\text { Er norm } \\
\mathrm{kV} / \mathrm{m}\end{array}$ \\
\hline 137.5 & 29.7 & $15 \mathrm{~cm}$ & 16.6 \\
\hline 142.5 & 15.7 & $20 \mathrm{~cm}$ & 8.77 \\
\hline 147.5 & 7.90 & $25 \mathrm{~cm}$ & 4.43 \\
\hline 152.5 & 4.02 & $30 \mathrm{~cm}$ & 2.25 \\
\hline 157.5 & 2.04 & $35 \mathrm{~cm}$ & 1.14 \\
\hline 162.5 & 1.03 & $40 \mathrm{~cm}$ & 0.577 \\
\hline 167.5 & 0.504 & $45 \mathrm{~cm}$ & 0.282 \\
\hline
\end{tabular}

\section{CURRENT DISTRIBUTION in the HOM COUPLER}

The real value of the current at specific locations in the HOM can now be calculated for a specified cavity gradient. Table 3 gives the field strength at a cavity voltage of 1 MV. Table 2 gives the current at field strength of $2.36 \mathrm{~V} / \mathrm{m}$. Combining them results in Table 4.

Table 4 The current at different points and different coupler location

\begin{tabular}{|c|c|c|c|c|c|c|c|}
\hline $\begin{array}{c}\text { HOM } \\
\text { location }\end{array}$ & T1-left & T1-right & T2-left & T2-right & L1 & L2 & Output \\
\hline $15 \mathrm{~cm}$ & $2.35 \mathrm{~A}$ & $2.83 \mathrm{~A}$ & $2.83 \mathrm{~A}$ & $2.00 \mathrm{~A}$ & $1.66 \mathrm{~A}$ & $7.24 \mathrm{~mA}$ & $1.41 \mathrm{~mA}$ \\
\hline $20 \mathrm{~cm}$ & $1.24 \mathrm{~A}$ & $1.49 \mathrm{~A}$ & $1.49 \mathrm{~A}$ & $1.06 \mathrm{~A}$ & $0.877 \mathrm{~A}$ & $3.83 \mathrm{~mA}$ & $0.75 \mathrm{~mA}$ \\
\hline $25 \mathrm{~cm}$ & $0.627 \mathrm{~A}$ & $0.755 \mathrm{~A}$ & $0.753 \mathrm{~A}$ & $0.533 \mathrm{~A}$ & $0.443 \mathrm{~A}$ & $1.93 \mathrm{~mA}$ & $0.38 \mathrm{~mA}$ \\
\hline $30 \mathrm{~cm}$ & $0.318 \mathrm{~A}$ & $0.383 \mathrm{~A}$ & $0.382 \mathrm{~A}$ & $0.271 \mathrm{~A}$ & $0.225 \mathrm{~A}$ & $0.98 \mathrm{~mA}$ & $0.19 \mathrm{~mA}$ \\
\hline $35 \mathrm{~cm}$ & $0.161 \mathrm{~A}$ & $0.194 \mathrm{~A}$ & $0.194 \mathrm{~A}$ & $0.137 \mathrm{~A}$ & $0.114 \mathrm{~A}$ & $0.50 \mathrm{~mA}$ & $0.097 \mathrm{~mA}$ \\
\hline $40 \mathrm{~cm}$ & $0.082 \mathrm{~A}$ & $0.098 \mathrm{~A}$ & $0.098 \mathrm{~A}$ & $0.069 \mathrm{~A}$ & $0.058 \mathrm{~A}$ & $0.25 \mathrm{~mA}$ & $0.049 \mathrm{~mA}$ \\
\hline $45 \mathrm{~cm}$ & $0.040 \mathrm{~A}$ & $0.048 \mathrm{~A}$ & $0.048 \mathrm{~A}$ & $0.034 \mathrm{~A}$ & $0.028 \mathrm{~A}$ & $0.12 \mathrm{~mA}$ & $0.024 \mathrm{~mA}$ \\
\hline
\end{tabular}

\section{TUNER SENSITIVITY}

As can be seen from Figs. 3 and 7, the notch is very sharp. This is advantageous, because the sharp shape means a high $\mathrm{Q}$ and implies that the fundamental frequency can be mostly depressed. On the other hand, it requires the frequency to be tuned precisely right on the fundamental frequency. From the viewpoint of mechanics one would prefer a moderate tuning rate so as to ease the tuner adjustments. It is then interesting to know the sensitivity of the tuner. 
The simulation based on the circuit model in Fig. 5 shows the tuning capacitance $\mathrm{C} 4$ as $3.00 \mathrm{pF}$, corresponding to a notch frequency of $709 \mathrm{MHz}$, very close to the fundamental frequency. On the other hand the measurement of the gap between the tuner and the inner conductor of the coupler, which tunes the notch to the fundamental frequency shown in Fig. 3, was determined to be 0.018 " or $0.45 \mathrm{~mm}$. Based on its geometrical shape, one can assume that the gap corresponds to $3 \mathrm{pF}$.

The capacitance $C$ is roughly inversely proportional to the gap. One has:

$$
\frac{d C}{d g}=-\frac{C}{g}
$$

If one ignores the sensitivity of the $\mathrm{T} 2$ inductance, then

$$
\frac{d f}{d C}=-\frac{f}{2 C}
$$

Therefore, one finds:

$$
\frac{d f}{d g}=\frac{f}{2 g}=\frac{709}{2 \times 0.45} \frac{\mathrm{MHz}}{\mathrm{mm}}=788 \mathrm{MHz} / \mathrm{mm}
$$

One can also check the sensitivity per PSpice simulation and finds the tuning rate to be

$$
d f / d C=-92 \mathrm{MHz} / \mathrm{pF} \text {. }
$$

Substituting (1), one gets

$$
d f / d g=613 \mathrm{MHz} / \mathrm{mm} \text {. }
$$

This result is lower because it takes into account the inductance change with frequency.

Certainly, tuning the frequency notch is very sensitive. If one wants to control the frequency within $1 \mathrm{MHz}$, the precision of the location of the tuner should be within one micron.

\section{CONCLUSION}

The measurement setup with a coaxial line is an appropriate arrangement to test the performance of a HOM coupler and to calculate easily the field strength acting on the coupler. The circuit model is a good approximation and easy to simulate. Once the field strength acting on the coupler in a real cavity assembly is known, which can be figured out by either $2 \mathrm{~d}$ or $3 \mathrm{~d}$ code, one can quantitatively determine the coupling and the current distribution. Finally, one can also find the tuner sensitivity, which would be important to specify the mechanical design requirements.

\section{ACKNOWLEDGEMENTS}

The design of the HOM coupler for the ERL superconducting cavity was provided by J. Sekutowicz. The coupler used for the present measurements was machined at Advanced Energy systems. The authors would like to acknowledge illuminating discussions with Jasek Sekutowicz and Dough Holmes. 